Eun-Kyeong Jo $\cdot$ Yue Wang

Hirokazu Kanegane · Takeshi Futatani

Chang-Hwa Song $\cdot$ Jeong-Kyu Park $\cdot$ Jung Soo Kim

Dong Soo Kim · Kang-Mo Ahn · Sang-Il Lee

Hyeon Jin Park · Youn Soo Hahn · Jae-Ho Lee

Toshio Miyawaki

\title{
Identification of mutations in the Bruton's tyrosine kinase gene, including a novel genomic rearrangements resulting in large deletion, in Korean X-linked agammaglobulinemia patients
}

Received: 17 March 2003 / Accepted: 8 April 2003 / Published online: 24 May 2003

(C) The Japan Society of Human Genetics and Springer-Verlag 2003

\begin{abstract}
Mutations in the Bruton's tyrosine kinase $(B T K)$ gene are responsible for X-linked agammaglobulinemia (XLA). We identified $B T K$ mutations in six patients with presumed XLA from unrelated Korean families. Four out of six mutations were novel: two missense mutations (P565T, C154Y), a point mutation in a splicing donor site (IVS11 $1 \mathrm{G}>\mathrm{A}$ ), and a large deletion (a 6.1-kb deletion including BTK exons 11-18). The large deletion, identified by long-distance PCR, revealed $A l u$-Alu mediated recombination extended from an $A l u$ sequence in intron 10 to another $A l u$ sequence in intron 18 , spanning a distance of $6.1 \mathrm{~kb}$. The two known mutations consisted of one missense (G462D) mutation,
\end{abstract}

E.-K. Jo · C.-H. Song · J.-K. Park

Department of Microbiology, College of Medicine,

Chungnam National University, Daejeon, Korea

Y. Wang $\cdot$ H. Kanegane $(\varangle) \cdot$ T. Futatani $\cdot$ T. Miyawaki

Department of Pediatrics, Faculty of Medicine,

Toyama Medical and Pharmaceutical University,

2630 Sugitani, Toyama 930-0194, Japan

E-mail: kanegane@ms.toyama-mpu.ac.jp

Tel.: + 81-76-4347313

Fax: + 81-76-4345029

J. S. Kim

Department of Pediatrics,

Chonbuk National University Medical School,

Jeonbuk, Korea

D. S. Kim

Department of Pediatrics, Yonsei University College of Medicine, Seoul, Korea

K.-M. Ahn · S.-I. Lee

Department of Pediatrics, Samsung Medical Center, Seoul, Korea

H. J. Park · Y. S. Hahn

Department of Pediatrics, College of Medicine,

Chungbuk National University, Chungbuk, Korea

J.-H. Lee

Department of Pediatrics, College of Medicine,

Chungnam National University, Daejeon, Korea and a point mutation in a splicing acceptor site (IVS7-9A $>$ G). This study suggests that large genomic rearrangements involving Alu repeats are few but an important component of the spectrum of $B T K$ mutations.

Keywords X-linked agammaglobulinemia · Bruton's tyrosine kinase - Mutation - Alu repeat recombination . Korea

\section{Introduction}

X-linked agammaglobulinemia (XLA), caused by mutations in Bruton's tyrosine kinase $(B T K)$, is the most common form of inherited antibody deficiency (Conley 1992). While pro-B cells in the bone marrow are present in normal or greater numbers, XLA-affected males have no or very low levels of circulating mature B cells (usually less than $1 \%$ of total lymphocytes), plasma cells, and immunoglobulins of all isotypes. The XLA patients suffer from recurrent, occasionally life-threatening infections from a very early age. The female carriers remain asymptomatic due to proliferation and maturation of $\mathrm{B}$ cells expressing the normal BTK gene product, as evidenced by the completely skewed $\mathrm{X}$ chromosome inactivation in their B cell lineage (Conley et al. 1986; Fearon et al. 1987).

$B T K$ is located on the long arm of the $\mathrm{X}$ chromosome at Xq22 and consists of 19 exons that span $37 \mathrm{~kb}$ of genomic DNA (Rohrer et al. 1994). BTK is composed of five distinct structural domains: the pleckstrin homology (PH), Tec homology (TH), Src homology 3 (SH3), SH2, and catalytic kinase (SH1) domains (Ohta et al. 1994; Sideras et al. 1994; Rohrer et al. 1994). A database of $B T K$ mutations lists more than 700 mutation entries from 667 unrelated families (http://bioinf.uta.fi/BTKbase). 
Mutations in $B T K$ are heterogeneous and spread throughout all five domains of BTK, and are associated with reductions in $B T K$ mRNA, protein, and kinase activity (Conley et al. 1994; Kornfeld et al. 1996; Tsukada et al. 1993; Saffran et al. 1994; Hashimoto et al. 1996; Bykowsky et al. 1996). Greater than $90 \%$ of BTK mutations can be identified using single-strand confirmation polymorphism (SSCP) screening of genomic DNA. However, the task of identifying larger genomic alterations may be formidable because of the lack of noncoding sequence data at the BTK locus needed to determine deletion breakpoints (Rohrer et al. 1999).

We describe here the BTK mutations found in six Korean patients with XLA. Four of them were novel, one of which was a deletion resulting from homologous recombination between $A l u$ repeats in introns 10 and 18 .

\section{Subjects and methods}

Subjects

The six male Korean hypogammaglobulinemic patients from six unrelated families enrolled in this study; none of them have been previously reported. All patients were male, aged between three and six years old, and had low levels of serum immunoglobulins. Clinical and immunological data of six patients are shown in Table 1.

The percentages of peripheral B cells were assessed by an immunofluorescence analysis using anti-CD19 monoclonal antibody (MoAb; Pharmingen, USA). All six patients showed markedly reduced B-cell numbers $(<1 \%)$, which implied that they had XLA.

\section{Analysis of BTK expression}

Expression of BTK protein in monocytes from the four patients (P2, P3, P4, and P5) was assessed by flow cytometric assay using anti-BTK monoclonal antibody as previously described. (Futatani et al. 1998).

\section{BTK mutation detection}

The $B T K$ mutations were detected by the direct sequencing of $B T K$ cDNA and by using polymerase chain reaction (PCR)-based single-strand conformation polymorphism (SSCP) analysis and direct sequencing of the corresponding exons, as described (Hashimoto et al. 1996; Jo et al. 2001; Kanegane et al. 2001). Total
RNA was extracted from PBMC with TRIzol Reagent (Life Technologies, Grand Island, N.Y.) and used for first-strand cDNA synthesis. PCR amplification of the cDNA involved seven overlapping primers (Hashimoto et al. 1996). The resulting PCR products were used as templates for direct sequencing by an automated ABI PRISM 310 DNA Genetic Analyzer (Applied Biosystems, Foster City, Calif.). Genomic DNA was purified from PBMC with a QIAamp Blood Kit (Qiagen, Hilden, Germany) and amplified by using primers encompassing each exon-intron boundary of the BTK gene (Vorechovsky et al. 1995). For SSCP analysis, the amplified products were electrophoresed on a Gene Excel 12.5/24 polyacrylamide gel (Amersham Pharmacia Biotech, Buckinghamshire, UK) for $90 \mathrm{~min}$ and were visualized by silver staining. When abnormal mobility was detected in the SSCP gel, the corresponding exons were sequenced as described above. Long-distance PCR of genomic DNA was performed with pairs of oligonucleotide primers specific to introns 9 and 19 of the $B T K$ gene (5'-ATCACTGACATGGACAAGCC-3' and 5'-TTGCTCAGAAGCCACTATCC- $3^{\prime}$ ). The reaction was carried out using Platinum Taq DNA Polymerase High Fidelity (Invitrogen, Carlsbad, Calif.).

\section{Results}

The clinical features of the six patients with XLA are shown in Table 1. Before intravenous immunoglobulin replacement, all patients except P5 had serum IgG levels $<300 \mathrm{mg} / \mathrm{dl}$, and the serum IgA and IgM levels were undetectable in some of these patients. A transient neutropenia (absolute neutrophil count, 462/ $\mu$ l) was observed in P6. All patients had recurrent bacterial infections, and all patients except $\mathrm{P} 3$ had pneumonia histories. However, they had no specific familial histories. Three patients $(\mathrm{P} 1, \mathrm{P} 3$, and $\mathrm{P} 5)$ had recurrent otitis media. The P5 suffered from various recurrent bacterial infections, including orbital cellulites, erysipelas, abscess, otitis media, sinusitis, and pneumonia. In addition, he had sepsis induced by Streptococcus pneumoniae infection. Currently, the six patients receive intravenous immunoglobulin replacement therapy, and are in apparently healthy states without any clinical symptoms.

Flow cytometric evaluation of BTK expression disclosed that BTK deficiency (1.7-14.1\%) was found in the four patients, suggesting that they were XLA patients (Table 2). Thereafter, we examined the BTK gene in patients with presumed XLA and the sequencing analysis detected BTK mutations in all six patients (Table 2). Among six XLA patients we studied, three

Table 1 Patient laboratory data and family history ( $y$ years, URTI upper respiratory tract infections)

\begin{tabular}{|c|c|c|c|c|c|c|c|c|}
\hline $\begin{array}{l}\text { Patient } \\
\text { no. }\end{array}$ & $\begin{array}{l}\text { Present } \\
\text { age }\end{array}$ & $\begin{array}{l}\text { Age of } \\
\text { onset }\end{array}$ & $\begin{array}{l}\text { Age at } \\
\text { diagnosis }\end{array}$ & \multicolumn{3}{|c|}{$\begin{array}{l}\text { Ig level } \\
(\mathrm{mg} / \mathrm{dl}) \text { at } \\
\text { onset }\end{array}$} & $\begin{array}{l}\text { Peripheral } \\
\text { B cells }(\%)\end{array}$ & Clinical presentation \\
\hline $\mathrm{P} 1$ & $6 \mathrm{y}$ & $4 \mathrm{y}$ & $4.5 \mathrm{y}$ & 70 & 35 & 15 & 0 & Pneumonisa, otitis media, sepsis \\
\hline $\mathrm{P} 2$ & $3 y$ & $2 y$ & $3 y$ & 227 & 33 & 7 & 1.0 & Frequent pneumonia \\
\hline P3 & $5 y$ & $3 y$ & $3 y$ & 192 & 5 & 7 & 0.1 & Sinusitis, otitis media, frequent URTI \\
\hline P6 & $3 y$ & $2 \mathrm{y}$ & $2 \mathrm{y}$ & 255 & 70 & 20 & 0 & Pneumonia \\
\hline
\end{tabular}


Table $2 B T K$ mutations and BTK protein expression identified in the present study (NT not tested)

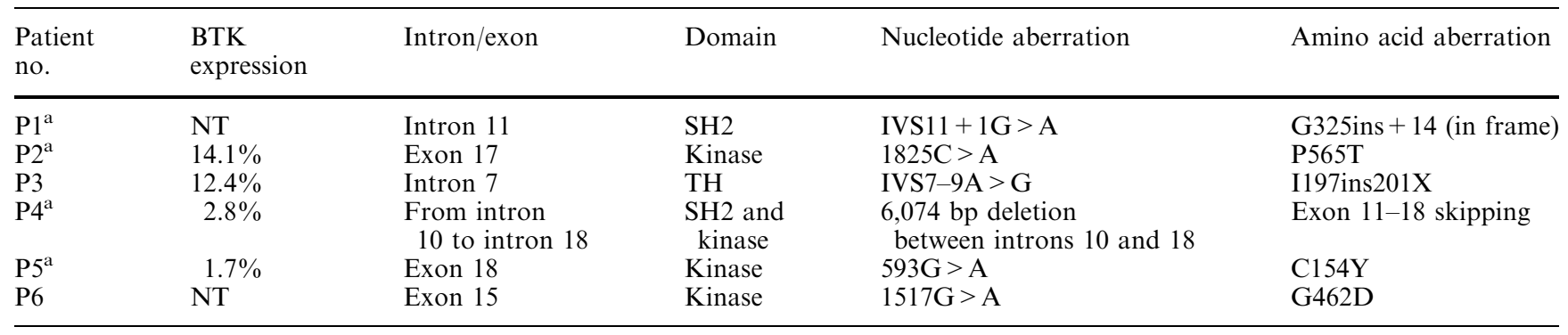

${ }^{\mathrm{a}}$ Novel mutations

(P2, P5, and P6) had missense mutations. P2 showed a C to A change at position 1825 , which resulted in a proline to threonine change at residue 565. The P565T mutation was a novel one, although a $\mathrm{C}$ to $\mathrm{T}$ point mutation at the same position was reported to cause a P565L substitution (Stewart et al. 2001). Another novel missense mutation, a $\mathrm{G}$ to A nucleotide change at position 593, was detected in P5, which resulted in a cysteine to tyrosine substitution at residue $154(\mathrm{C} 154 \mathrm{Y})$. In the previous report, the mutation $593 \mathrm{G}>\mathrm{C}$ caused a cysteine to serine substitution (Vihinen et al. 1997). In P6, the $1517 \mathrm{G}>\mathrm{A}$ aberration caused the amino-acid change G462D, as previously submitted to the BTKbase (http:// bioinf.uta.fi/BTKbase).

Two splicing errors were found in the genetic material of patients $\mathrm{P} 1$ and $\mathrm{P} 3$. A point mutation was found in intron $11(+1 \mathrm{G}>\mathrm{A})$ by sequencing in the genomic DNA of P1. The IVS11 + $1 \mathrm{G}>\mathrm{A}$ usually causes exon 11 skipping; however, P1 showed an insertion of $42 \mathrm{bp}$ at the $5^{\prime}$ end of intron 11 [G325ins +14 (in frame)]. In this case, intron $11+43+44$ gt might be a new splice acceptor site. Although a previous report demonstrated the first $\mathrm{G}$ nucleotide deletion at the splice donor site for intron 11 (Conley et al. 1994), this is a novel splicing mutation at the exon 11/intron 11 border. The other splicing change in P3 was found at the -9 position of intron $7(-9 A>G)$, which created an aberrant splicing receptor site. This change resulted in the addition of a novel exon-like sequence of 8 bp between exons 7 and 8 . The resulting frameshift introduced a protein termination signal at codon 201 (I197ins201X). The same mutation was already submitted to the BTKbase (http:// bioinf.uta.fi/BTKbase); however, the current study confirms the resulting amino acid change of this genetic alteration.

The final mutation was found to be a $6.1-\mathrm{kb}$ deletion including $B T K$ exons $11-18$. To better understand the mechanism by which this mutation had occurred, we characterized this alteration in detail. Analysis of P4 genomic DNA by a PCR method showed that there is no product from exons 11 to 18 . Since PCR amplification for exons 10 and 19 were normal, the sense primer for the exon 10 boundary and the anti-sense primer for the exon 19 boundary were used to PCR-amplify the patient's genomic DNA. These primers, normally separated by $9.9 \mathrm{~kb}$ of genomic DNA, specifically amplified

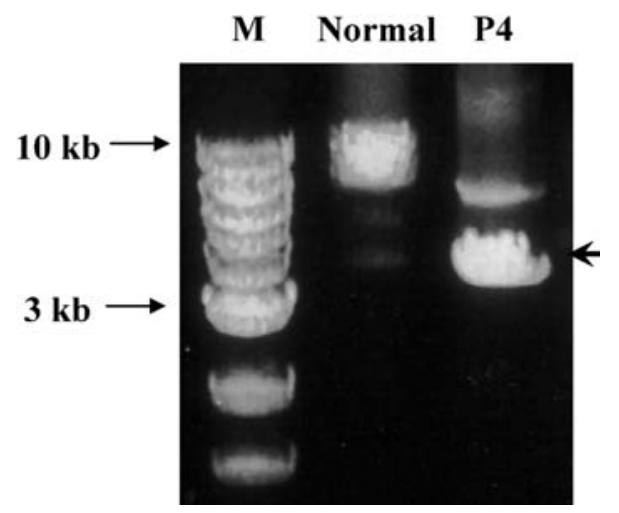

Fig. 1 Long-distance PCR of genomic DNA from P4. PBMCs were isolated from P4, and normal control and genomic DNAs were purified as described in the text. Long-distance PCR of genomic DNA from P4 was performed with pairs of oligonucleotide primers specific to introns 9 and 19 of the BTK gene. An arrowhead shows the deletion of $6.1 \mathrm{~kb} ; M$ standard size markers

a 3.8-kb fragment when using patient DNA (Fig. 1). Sequence analysis of the PCR product revealed that an inverted Alu sequence repeat (position 62318-62619; U78027) within intron 10 had homologously recombined with an inverted Alu sequence repeat (position 68391-68559; U78027), resulting in the formation of a new full-length $A l u$ and the deletion of $6.1 \mathrm{~kb}$ (Fig. 2).

In conclusion, we described here the molecular identification of six XLA patients in Korea. Our data emphasize that initial characterization of $B T K$ mutations - not readily detectable by PCR-SSCP analysis should include a search for Alu and other repetitive elements to facilitate subsequent PCR-based mutation analysis. Further identifications of BTK mutations should be compiled and analyzed to provide data for clarifying correlation between the severity of the disease and the type and location of the mutations found in XLA patients.

\section{Discussion}

Although more than 700 BTK mutations have been reported in the patients with XLA (http://bioinf.uta.fi/ BTKbase), most alterations in $B T K$ are single base-pair 
Fig. 2 Homologous sequences from patient DNA and Alu repeat of introns 10 and 18 Sequence data of the PCR product from $\mathrm{P} 4$ show homologies with $A l u$ sequence repeats in introns 10 and 18 of the $B T K$ gene. The nucleotide numbers were designated by GenBank accession U78027
Intron 1062279 GTCAAAGC TGCCATCG TCTGGGATCCCAAT TACACCATTTTTTTTGTTTTTGTTTTTCTG Patient GTCAAAGCTGCCATCGTCTGGGATCCCAATTACACCATTTTTTTGTTTTGTTTTCTG

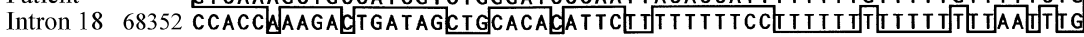

Intron 1062339 AGACGGACTCTCGGTC TGTCACCCAGGCTGGAG TGCAGTGGCG GATCTGGGCTCACTGC Patient AGACGGACTCTCGGTCTGTCACCCAGGCTGGAGTACAGTGGCGCGATCTTGGCTTACTGC Intron 1868412 AGACAAGG TCGCTCTTCACCCAGGTGGAGTACAGTGGCGCGATCTTGGCTTACTGC

Intron 1062399 AACC TCCGCCTTCT GG TTCAAGTA ATTCTCC Patient AACCTTCGCCTCCCAGATTCAAGCGATTCTCATGTCTCAGCCTCCCAAGTAGCTGGGATT Intron 1868472 AACCTTCGCCTCCCAGATTCAAGCGATTCTCATGTCTCAGCCTCCCAAGTAGCTGGGATT

Intron 1062459 ACAGGCACATGCCACCACGCCCGACTAATTTTTGTATTTTTAGCAFAGACGGGGTTCAC Patient Intron 1868532 ATAGGCGTGCACCACCACACCAAGCTAAT TT TTTTTTTTTTTTGGTGACACAGTCT TG substitutions or small insertions or deletions that are readily detectable by PCR-SSCP analysis. However, some mutations are not readily detectable by conventional SSCP analysis. Particular genetic alterations, such as retroposon insertion, inversion, duplication, and large deletions could be detected by large-scale sequencing. The repetitive DNA elements, including Alu repeats, played a significant role in the underlying molecular mechanisms in these alterations (Rohrer et al. 1999). Human Alu elements play a major role in genome remodeling, both by de novo insertion and by promoting deletions and re-duplications as a result of unequal recombination between dispersed repeat elements (Deininger and Batzer 1999). Alu repeats are composed of two internal tandem repeats - a left arm, $132 \mathrm{bp}$ in length, and a right arm, 159 bp long (Deininger et al. 1981) - and have reached a copy number in excess of 500,000 copies per human genome. These elements have been proposed to have a number of functions in the human genome, and have certainly had a major impact on genomic architecture (Deininger and Batzer 1999). The frequency of mutagenesis caused by unequal homologous recombination between Alu repeats is estimated as $0.3 \%$ of human genetic diseases, and Alu elements continue to contribute to a significant portion of human genetic diseases (Deininger and Batzer 1999). To our knowledge, this is the fifth mutation involving $A l u$ repeat recombination in $B T K$ gene.

Acknowledgements We are very grateful to all the families for their generous cooperation in this study. We also thank Ms. Chikako Sakai for the excellent technical assistance. This study was financially supported by the Korea Science and Engineering Foundation in the 2002 program year (F01-2002-000-20026-0), the Ministry of Education, Culture, Sports, Science and Technology of Japan, and the Ministry of Health, Labour and Welfare of Japan.

\section{References}

Bykowsky MJ, Haire RN, Ohta Y, Tang H, Sung S-SJ, Veksler ES, Greene JM, Fu SM, Litman GW, Sullivan KE (1996) Discordant phenotype in siblings with $\mathrm{X}$-linked agammaglobulinemia. Am J Hum Genet 58:477-483

Conley ME (1992) Molecular approaches to analysis of X-linked immunodeficiencies. Annu Rev Immunol 10:215-238

Conley ME, Brown P, Pickard AR, Buckley RH, Miller DS, Raskind WH, Singer JW, Fialkow PJ (1986) Expression of the gene defect in X-linked agammaglobulinemia. N Engl J Med 315:564-567
Conley ME, Fitch-Hilgenberg ME, Cleveland JL, Parolini O, Rohrer J (1994) Screening of genomic DNA to identify mutations in the gene for Bruton's tyrosine kinase. Hum Mol Genet 3:1751-1756

Deininger PL, Batzer M (1999) Alu repeats and human disease. Mol Genet Metab 67:183-193

Deininger PL, Jolly DJ, Rubin CM, Friedmann T, Schmid CW (1981) Base sequence studies of 300 nucleotide renatured repeated human DNA clones. J Mol Biol 151:17-33

Fearon ER, Winkelstein JA, Civin CI, Pardoll DM, Vogelstein B (1987) Carrier detection in X-linked agammaglobulinemia by analysis of X-chromosome inactivation. N Engl J Med 316:427431

Futatani T, Miyawaki T, Tsukada S, Hashimoto S, Kunikata T, Arai S, Kurimoto M, Niida Y, Matsuoka H, Sakiyama Y, Iwata T, Tsuciya S, Tatsuzawa O, Yoshizaki K, Kishimoto T (1998) Deficient expression of Bruton's tyrosine kinase in monocytes from X-linked agammaglobulinemia as evaluated by a flow metric analysis and its clinical application to carrier detection. Blood 91:595-602

Hashimoto S, Tsukada S, Matsushita M, Miyawaki T, Niida Y, Yachie A, Kobayashi S, Iwata T, Hayakawa H, Matsuoka H, Tsuge I, Yamadori T, Kunikata T, Arai S, Yoshizaki K, Taniguchi N, Kishimoto T (1996) Identification of Bruton's tyrosine kinase (Btk) gene mutations and characterization of the derived proteins in $35 \mathrm{X}$-linked agammaglobulinemia families: a nationwide study of Btk deficiency in Japan. Blood 88:561-573

Jo EK, Kanegane H, Nonoyama S, Tsukada S, Lee JH, Lim K, Shong M, Song CH, Kim HJ, Park JK, Miyawaki T (2001) Characterization of mutations, including a novel regulatory defect in the first intron, in Bruton's tyrosine kinase gene from seven Korean X-linked agammaglobulinemia families. J Immunol 167:4038-4045

Kanegane H, Futatani T, Wang Y, Nomura K, Shinozaki K, Matsukura H, Kubota T, Tsukada S, Miyawaki T (2001) Clinical and mutational characteristics of X-linked agammaglobulinemia and its carrier identified by flow cytometric assessment combined with genetic analysis. J Allergy Clin Immunol 108:1012-1020

Kornfeld SJ, Haire RN, Strong SJ, Tang H, Sung S-SJ, Fu SM, Litman GW (1996) A novel mutation (Cys145stop) in Bruton's tyrosine kinase is associated with newly diagnosed X-linked agammaglobulinemia in a 51-year-old male. Mol Med 2:619623

Ohta Y, Haire RN, Litman RT, Fu SM, Nelson RP, Kratz J, Kornfeld SJ, de la Morena M, Good RA, Litman GW (1994) Genomic organization and structure of Bruton agammaglobulinemia tyrosine kinase: localization of mutations associated with varied clinical presentations and course in X chromosomelinked agammaglobulinemia. Proc Natl Acad Sci USA 91:90629066

Rohrer J, Parolini O, Belmont JW, Conley ME (1994) The genomic structure of human BTK, the defective gene in X-linked agammaglobulinemia. Immunogenetics 40:319-324

Rohrer J, Minegishi Y, Richter D, Eguiguren J, Conley ME (1999) Unusual mutations in Btk: an insertion, a duplication, an inversion, and four large deletions. Clin Immunol 90:28-37 
Saffran DC, Parolini O, Fitch-Hilgenberg ME, Rawlings DJ, Afar DEH, Witte ON, Conley ME (1994) Brief report: A point mutation in the $\mathrm{SH} 2$ domain of Bruton's tyrosine kinase in atypical X-linked agammaglobulinemia. N Engl J Med 330:1488-1491

Sideras P, Muller S, Shiels H, Jin H, Khan WN, Nilsson L, Parkinson E, Thomas JD, Branden L, Larsson I, Paul WE, Rosen FS, Alt FW, Vetrie D, Smith CIE, Xanthopoulos G (1994) Genomic organization of mouse and human Bruton's agammaglobulinemia tyrosine kinase (Btk) loci. J Immunol 153: $5607-5617$

Stewart DM, Tian L, Nelson DL (2001) A case of X-linked agammaglobulinemia diagnosed in adulthood. Clin Immunol 99:94-99
Tsukada S, Saffran DC, Rawlings DJ, Parolini O, Allen RC, Klisak I, Sparkes RC, Kubagawa H, Mohandas T, Quan S, Belmont JW, Cooper MD, Conley ME, Witte ON (1993) Deficient expression of a B cell cytoplasmic tyrosine kinase in human X-linked agammaglobulinemia. Cell 72:279-290

Vihinen M, Nore BF, Mattsson PT, Backesjo CM, Nars M, Koutaniemi S, Watanabe C, Lester T, Jones A, Ochs HD, Smith CI (1997) Missense mutations affecting a conserved cysteine pair in the TH domain of Btk. FEBS Lett 413:205-210

Vorechovsky I, Vihinen M, de Saint Basile G, Honsova S, Hammarstrom L, Muller S, Nilsson L, Fischer A, Smith CI (1995) DNA-based mutation analysis of Bruton's tyrosine kinase gene in patients with X-linked agammaglobulinaemia. Hum Mol Genet 4:51-58 